\title{
Adesão às precauções-padrão entre profissionais da enfermagem expostos a material biológico*
}

\author{
Adherence to standard precautions among nursing professionals exposed to biological material
}

Adhesión a las precauciones estándar entre profesionales de enfermería expuestos a material biológico

Silmara Elaine Malaguti-Toffano ${ }^{1}$, Silvia Rita Marin da Silva Canini ${ }^{2}$, Renata Karina Reis ${ }^{3}$, Fernanda Maria Vieira Pereira ${ }^{4}$, Adriana Maria da Silva Felix ${ }^{5}$, Patricia Helena Vivan Ribeiro ${ }^{6}$, Elucir Gir $^{7}$

* Extraído da Tese de Doutorado intitulada "Adesão às Precauções-Padrão de profissionais de enfermagem de um hospital universitário" apresentada à Escola de Enfermagem de Ribeirão Preto da Universidade de São Paulo, em 2011.

${ }^{1}$ Enfermeira, Doutora em Enfermagem. Professora Adjunta da Universidade Federal de São João Del-Rei. Divinópolis, MG, Brasil. E-mail: silmalaguti@yahoo.com.br.

${ }^{2}$ Enfermeira, Doutora em Enfermagem. Professora Associada da Escola de Enfermagem de Ribeirão Preto da Universidade de São Paulo (EERP/USP). Ribeirão Preto, SP, Brasil. E-mail: canini@eerp.usp.br.

${ }^{3}$ Enfermeira, Doutora em Enfermagem Fundamental. Professora Doutora da EERP/USP. Ribeirão Preto, SP, Brasil. E-mail: rkreis@eerp.usp.br.

${ }^{4}$ Enfermeira, Mestre em Enfermagem Fundamental. Discente do Programa Interunidades de Doutoramento em Enfermagem da Escola de Enfermagem/USP e da Escola de Enfermagem de Ribeirão Preto/USP. Ribeirão Preto, SP, Brasil. E-mail: fernanddamaria@hotmail.com.

${ }^{5}$ Enfermeira, Doutora em Enfermagem. Ribeirão Preto, SP, Brasil. E-mail: adrianausprp@yahoo.com.br.

${ }^{6}$ Enfermeira, Doutora em Enfermagem. Ribeirão Preto, SP, Brasil. E-mail: patriciavivan@sercontel.com.br.

${ }^{7}$ Enfermeira, Doutora em Enfermagem. Professora Titular da EERP/USP. Ribeirão Preto, SP, Brasil. E-mail: egir@eerp.usp.br.

\section{RESUMO}

Estudo transversal, realizado em hospital público de ensino, com 256 profissionais de enfermagem, cujos objetivos foram descrever as exposições ocupacionais envolvendo material biológico, potencialmente contaminado, entre profissionais de enfermagem de um hospital universitário e comparar os escores de adesão às medidas de Precauções-Padrão (PP). Na primeira etapa da coleta de dados, utilizou-se um formulário com questões referentes à exposição ocupacional e à escala psicométrica "Adesão às PP"; na segunda, a partir do consentimento dos participantes, foi realizada consulta em prontuários dos profissionais expostos. A adesão às PPs foi avaliada como alta e intermediária, segundo as respostas dos itens da escala. Não houve diferença estatisticamente significante entre os expostos ou não ao material biológico. Evidenciou-se que somente o uso da escala de adesão às PPs não foi suficiente para contemplar todos os fatores que podem estar associados à exposição ocupacional com material biológico.

Descritores: Precauções Universais; Riscos Ocupacionais; Acidentes de Trabalho; Enfermagem; Exposição Ocupacional.

\section{ABSTRACT}

This cross-sectional study, developed at a public teaching hospital with 256 nursing professionals, had the aim to: describe occupational exposure events involving potentially infectious biological material among nursing professionals at a teaching hospital and compare the scores of adherence to Standard Precautions (SP). The first stage of data collection was performed using a form with questions regarding the occupational exposure and the psychometric scale "Adherence to SP"; in the second stage, and upon the participants' consent, the medical records of the exposed professionals were analyzed. Adherence to SP was assessed as high and intermediate, according to the answers to the scale items. There was no statistically significant difference between those who had been exposed or not to biological material. The results evidence that only the use of the scale of adherence to SP was not enough to contemplate all factors that may be associated with occupational exposure to biological material.

Descriptors: Universal Precautions; Occupational Risks; Accidents, Occupational; Occupational Exposure.

\section{RESUMEN}

Estudio transversal, realizado en hospital público de enseñanza, con 256 profesionales de enfermería, objetivando describir las exposiciones laborales a material biológico potencialmente contaminado entre profesionales de un hospital universitario, y comparar los puntajes de adhesión a las medidas de Precauciones-Estándar (PE). Datos recolectados en primera etapa mediante formulario con preguntas referentes a exposición laboral y escala psicométrica "Adhesión a PE"; y en la segunda, con consentimiento de los participantes, se efectuó consulta de historias clínicas de los profesionales expuestos. La adhesión a las PE fue evaluada como alta y media, según las respuestas a los ítems de la escala. No existió diferencia estadísticamente significativa entre los expuestos o no al material biológico. Se evidenció que solamente el uso de la escala de adhesión a las PE no fue suficiente para contemplar todos los factores asociados a la exposición laboral a material biológico.

Descriptores: Precauciones Universales; Riesgos Laborales; Accidentes de Trabajo; Exposición Profesional. 


\section{INTRODUÇÃO}

A exposição ocupacional envolvendo material biológico potencialmente contaminado constitui um risco para os profissionais da área da saúde, sobretudo-àqueles da enfermagem que prestam cuidado direto aos pacientes e manuseiam, frequentemente, objetos cortantes e fluidos corporais.

Vários patógenos podem ser transmitidos aos profissionais de saúde ${ }^{(1)}$, em decorrência de suas atividades laborais, sendo os vírus da Imunodeficiência Humana (HIV), das hepatites B (VHB) e C (VHC) os de maior relevância epidemiológica.

No Brasil, o registro do primeiro caso de infecção ocupacional pelo HIV ocorreu em 1994, com uma auxiliar de enfermagem e decorreu da exposição percutânea envolvendo sangue ${ }^{(2)}$ e outros quatro casos de transmissão do HIV relativamente a profissionais de enfermagem foram documentados em pesquisas e tiveram como características comuns a exposição percutânea envolvendo sangue ${ }^{(3-4)}$.

Profissionais de enfermagem são descritos na literatura como os mais expostos ao acidente envolvendo material biológico ${ }^{(2-6)}$, principalmente, em situações de exposições percutâneas, quando da punção venosa e administração de medicamentos ${ }^{(7-10)}$.

Com o intuito de minimizar os riscos de exposição ocupacional a material biológico potencialmente contaminado, várias medidas de segurança vêm sendo estabelecidas nos serviços de saúde, entre elas as Precauções-Padrão (PP), ou seja, um conjunto de medidas de prevenção primária eficaz para reduzir o risco de transmissão de patógenos veiculados pelo sangue e por fluídos corporais ${ }^{(11)}$.

As medidas de PP aplicam-se a qualquer paciente, independentemente, do diagnóstico clínico ou sorológico e equipamentos de proteção individual (EPI), como luvas de procedimento, aventais, máscaras cirúrgicas e protetores oculares são recomendados sempre que o contato com fluidos corporais for previsto. Reforçam, tais medidas, a recomendação de higienização das mãos, antes e após o contato com pacientes e fluidos orgânicos, o descarte de materiais perfurocortantes em recipientes rígidos, cuidados com reprocessamento de materiais e administração de medicamentos injetáveis ${ }^{(11-12)}$.

Apesar de as medidas de PP serem apontadas, pela comunidade científica, como uma das mais importantes e eficazes medidas preventivas pré-exposição a material biológico, ainda há baixa adesão por parte dos profissionais da área da saúde (13-14). $^{\text {. }}$
Diante deste contexto o presente estudo teve como objetivos comparar os escores de adesão às PP entre os profissionais da equipe de enfermagem que sofreram ou não exposição ocupacional em um hospital de ensino e descrever as características das-dessas exposições quanto ao tipo, objeto, fluído corporal envolvido e uso de EPI no momento do acidente.

\section{MÉTODO}

Trata-se de um estudo de corte transversal, realizado no período de 2009 a 2010, em um hospital de ensino de grande porte do interior paulista.

A população de referência contava com 590 profissionais de enfermagem, lotados nas unidades de Clínica Médica, Cirúrgica, Ginecologia e Centro de Terapia Intensiva (CTI). Por meio de cálculo amostral ( $a=0,01$; effect size $=0,08$, poder do teste igual a 0,99 ) estimouse 290 sujeitos. Neste estudo, participaram 256 (100\%) profissionais, sendo $178 \quad(69,5 \%)$ auxiliares de enfermagem, 51 (19,9\%) enfermeiros e 27 (10,5\%) técnicos de enfermagem, com perda de $11,7 \%$.

Considerou-se como critérios de inclusão atuar no mínimo há seis meses na instituição, realizar procedimentos que envolvam fluidos corporais e no caso de enfermeiros, ser enfermeiro assistencial, função descrita para o primeiro nível hierárquico do enfermeiro no hospital de estudo. E como critérios de exclusão, profissionais que realizam, exclusivamente, atividades burocráticas, estar em licença-saúde indeterminada ou afastado por quaisquer outros motivos no período de coleta de dados.

Para a primeira etapa da coleta de dados foi aplicado um formulário semiestruturado contendo variáveis demográficas, como sexo, idade, categoria profissional e as seguintes questões fechadas "Sofreu exposição ocupacional com material biológico nos últimos anos? Buscou atendimento médico?" e ainda, a escala psicométrica Escala de "Adesão às Precauções-Padrão"(15) a todos os participantes, durante o turno de trabalho, em momentos mais oportunos.

A Escala de "Adesão às Precauções-Padrão" foi traduzida e validada para o Brasil ${ }^{(15)}$ por meio de adaptação transcultural e, mediante autorização dos autores, foi aplicada aos sujeitos desta investigação. Essa escala é do tipo Likert e é composta por 13 itens e cada um com alternativas que variam de 1 a 5 pontos.

A escala possui itens específicos quanto ao uso de EPI, como luvas descartáveis, óculos de proteção e avental que são itens avaliados na Escala de "Adesão às PP" e ainda, um escore total onde os níveis de adesão às PP são 
calculados segundo a média dos escores médios simples de cada item e classificados em: a) alto: para escores médios iguais ou superiores a 4,5; b) intermediário: para escores médios com valores entre 3,5 e 4,49 e c) baixo: para escores médios abaixo de $3,5^{(15)}$.

A segunda etapa foi composta pela consulta aos prontuários dos profissionais da enfermagem para identificar as variáveis da exposição ocupacional a material biológico, como tipo de exposição, objeto envolvido e uso de EPI no momento do acidente.

O estudo piloto foi realizado com 95 profissionais da enfermagem que atuavam em outros setores do hospital, local de realização do estudo.

As variáveis do instrumento foram codificadas e catalogadas em um dicionário (codebook). Os dados foram duplamente digitados em planilha Excel for Windows 2003 e após correção dos erros de digitação foram transportados para o Statistical Package for the Social Sciences (SPSS), versão 15.0.

Realizou-se análise estatística descritiva para caracterização da amostra e das exposições ocupacionais e os seguintes testes estatísticos: a) alfa de Cronbach; Kolmogorov-Smirnov, para testar a normalidade da distribuição das médias dos escores da escala em grupos com número de sujeitos inferior a 30; c) ANOVA (Analyses of Variance), para analisar a diferença entre os escores médios de adesão às PP entre as categorias profissionais de enfermagem e d) Teste t de Student para comparar os escores de adesão às PP e exposição a material biológico.

O projeto de pesquisa foi aprovado pelo Comitê de Ética em Pesquisa do Hospital das Clínicas da Faculdade de Medicina de Ribeirão Preto da Universidade de São Paulo (Protocolo 4620/2009) e os aspectos éticos foram contemplados. Todos os participantes concordaram em participar das duas etapas de coleta de dados.

\section{RESULTADOS}

Dos 256 profissionais participantes do presente estudo 69,6\% eram auxiliares de enfermagem, 10,5\% técnicos de enfermagem e 19,9\% enfermeiros. Houve predomínio do sexo feminino $(78,9 \%)$, com idade média de 38,6 anos (variação de 21,3 a 60,4 anos). A maioria possuía ensino médio completo $(64,5 \%)$.

A análise dos escores da escala de adesão às PP apontou que $59,4 \%$ dos profissionais apresentaram escores médios altos, ou seja, igual ou acima de 4,5; para $38,3 \%$ profissionais o escore médio foi intermediário, entre 3,5 e 4,49 e 2,3\% obtiveram escores baixos, ou seja, menores que 3,5. A análise de confiabilidade da escala de adesão às PP teve resultado geral igual a 0,70 e foi considerada satisfatória.

Tabela 1: Escore médio, desvio padrão, erro padrão médio da Escala de Adesão às Precauções-Padrão* dos profissionais de enfermagem que referiram não ter sofrido exposição ocupacional a material biológico e que tiveram registros de exposição no prontuário. Ribeirão Preto, SP, Brasil, 2009-2010.

\begin{tabular}{cccc}
\hline Registro de exposição Ocupacional & Média & Desvio Padrão & Erro padrão médio \\
\hline $\operatorname{Sim}(n=25)$ & 4,516 & 0,413 & 0,08279 \\
Não $(n=187)$ & 4,524 & 0,413 & 0,03020 \\
\hline
\end{tabular}

* (Brevidelli; Cianciarullo, 2009)

Os profissionais que se expuseram ou não ao material biológico potencialmente contaminado foram categorizados de acordo com as suas respostas e registros de prontuários, sendo então realizada a análise dos escores entre os grupos.

Os participantes que inicialmente, seriam divididos em dois grupos (expostos ou não a material biológico), foram divididos em quatros grupos, sendo: Aprofissionais que responderam ter sofrido exposição ocupacional, nos últimos dois anos, quando assim responderam no instrumento de coleta de dados e que também tiveram registros no prontuário $(n=18)$, Bprofissionais que referiram - no instrumento de coleta de dados - ter sofrido exposição, mas não tiveram registros nos prontuário $(n=26), C$ - profissionais que não referiram exposição no instrumento de coleta de dados, mas tiveram registro no prontuário $(n=25)$ e $D$ - não relataram exposição e não tiveram registro nos prontuários $(n=187)$.

$\mathrm{Na}$ comparação entre o grupo A e B, verificou-se distribuição normal das médias dos escores da escala de adesão nos profissionais do grupo $A(p=0,994)$ e $B$ $(p=0,876)$. Foi identificada diferença nos escores de adesão às $\operatorname{PP}(p=0,587)$ entre os profissionais do grupo $A$ (média =4,594; $d p=0,287$ ) e $B \quad$ (média=4,527; $\mathrm{dp}=0,321$ ), porém não se observaram diferenças entre os dois grupos ( $t$ student $\mathrm{p}=0,587$ ).

Na comparação entre o grupo C e $D$, também houve distribuição normal para o grupo $C(p=0,755)$. Não houve diferença dos escores de adesão às PP no grupo $C$ (média $=4,516 ; d p=0,41$ ) e $D$ (média $=4,524 ; p=0,41$ ) assim como não houve diferenças entre os dois grupos ( $t$ student $\mathrm{p}=0,6787)$. 
Quanto às exposições ocupacionais, segundo os registros, $44(17,2 \%)$ trabalhadores sofreram 52 exposições ocupacionais com material biológico nos últimos dois anos, sendo $80,7 \%$ percutâneas, 17,4\% mucocutâneas e 1,9\% contato com pele não íntegra. A agulha com lúmen foi o objeto envolvido em $77,0 \%$ e o sangue também esteve presente em $77,0 \%$ exposições.

Ressalta-se que $32,7 \%$ das exposições se efetivaram durante punção venosa; $11,5 \%$ na glicosimetria; $5,7 \%$ no reencape de agulhas; $3,8 \%$ no descarte de perfucortantes; $3,8 \%$ na troca de soro/equipo e 3,8\% no manuseio de material cirúrgico. Em relação à utilização de equipamento de proteção individual, em 53,8\% das exposições, os profissionais não utilizavam luvas no momento do acidente. Em 88,4\% das exposições, os profissionais não utilizavam óculos de proteção. Não foram identificadas informações sobre o uso de avental nos prontuários.

Entre as exposições, 42,3\% envolveram agulhas com lúmen, contendo sangue e em $23 \%$ os profissionais não estavam utilizando luvas de procedimentos. A quimioprofilaxia foi indicada em $34,6 \%$ das exposições. Destaca-se que não foi verificado nenhum caso de soroconversão, segundo dados obtidos nas fichas de atendimento do ambulatório especializado.

\section{DISCUSSÃo}

A análise dos escores da escala de adesão às PP apontou que $59,4 \%$ dos profissionais apresentaram escores médios altos e 38,3\% profissionais o escore médio foi intermediário e apenas 2,3\% baixo. Na análise da média dos itens individuais da escala foi identificado que $87,9 \%$ dos profissionais responderam que "sempre" utilizam as luvas em procedimentos que envolvam material biológico, porém observou-se nos registros de exposição, que o EPI não foi usado no momento do acidente.

Um estudo realizado nas unidades de terapia intensiva da mesma instituição, com profissionais de enfermagem, obteve escore médio intermediário para a escala de "Adesão às PP", no entanto, os autores observaram que itens importantes da escala não tiveram adesão em sua totalidade, como o descarte adequado de materiais perfurocortantes, a higienização das mãos após retirar luvas descartáveis e a utilização de óculos protetores $^{(14)}$.

No presente estudo, a maioria das exposições foi percutânea $(80,7 \%)$ durante punção venosa e realização do teste de glicosimetria. Uma investigação brasileira evidenciou que tais agulhas estiveram envolvidas em exposições percutâneas ocorridas em um hospital, porém, com a introdução de uma lanceta descartável e retrátil, houve queda significativa de exposições percutâneas por agulhas de pequeno calibre entre os profissionais de enfermagem ${ }^{(16)}$.

Do total de exposições percutâneas, 3,8\% ocorreram no momento do descarte e $9,5 \%$ por reencape de agulhas com lúmen ou com introdutor de cateter periférico. Tais ocorrências também foram evidenciadas por outras investigações que envolveram profissionais da enfermagem $^{(17-20)}$.

Segundo dados referentes a 62.970 notificações de exposições com material biológico do estado de São Paulo, o uso de luvas de procedimento durante o momento do acidente ocorreu em $74,4 \%$ das vezes ${ }^{(7)}$. Nesse mesmo estudo, observou-se que estas foram usadas somente em $35,4 \%$ dos casos de administração de medicamentos e punções venosas para coleta de sangue, bem como em $18,9 \%$ dos casos envolvendo a coleta de sangue ${ }^{(7)}$.

Em relação aos óculos de proteção, nos registros notou-se que em todas as exposições mucocutâneas, os mesmos não foram utilizados.

No presente estudo, alguns profissionais informaram no instrumento de coleta de dados que sofreram exposições, mas não buscaram atendimento médico, constatado também pelos registros nos prontuários. Isso também foi constatado em outros estudos brasileiros em que profissionais de enfermagem expostos não buscaram atendimento médico ou não notificaram os acidentes ${ }^{(21-22)}$. Uma investigação realizada na mesma instituição apontou que $29,2 \%$ dos acidentes não foram notificados oficialmente e que muitos profissionais que buscaram atendimento médico no ambulatório não procuraram o Serviço de Engenharia, Segurança e Medicina do Trabalho para a notificação oficial da ocorrência.

\section{CONCLUSÃO}

Profissionais de enfermagem sofreram exposições percutâneas e mucocutâneas. Identificou-se subnotificação de exposições ao verificar que profissionais que responderam ter sofrido exposição ocupacional com material biológico não tinham registros de acidentes em seus prontuários. Os dados evidenciaram adesão alta e intermediária quanto aos escores totais da Escala de adesão às PP, no entanto, não houve diferenças entre os participantes expostos ou não a material biológico.

Evidenciou-se no presente estudo que somente o uso da escala de adesão às PP não foi suficiente para contemplar todos os fatores que podem estar associados à exposição ocupacional com material biológico. Reforça- 
se assim, a necessidade de outras pesquisas, como estudos observacionais, que possam comparar os escores de adesão às PP obtidos pela aplicação da escala de

\section{REFERÊNCIAS}

1. Tarantola A, Abiteboul D, Rachline A. Infection risks following accidental exposure to blood or body fluids in health care workers: A review of pathogens transmitted in published cases. Am J Infect Control. 2006;34(6):367-75.

2. Santos NJS, Monteiro ALC, Ruiz EAC. The first case of due to occupational in Brazil. Brazian. Jour. Infect. Disease. 2002;

6(3):140-1.

3. Rapparini C. Occupational HIV infection among health care workers exposed to blood and body fluids in Brazil. Am J Infect Control. 2006;34(4):237-40.

4. Lucena NO, Pereira FR, Barros FS, Silva NB, Alexandre MAA, Castilho MC, et al. Infecção pelo HIV-1 após acidente ocupacional, no Estado do Amazonas: primeiro caso documentado. Revista Soc Bras Med Tropical. 2011;44(5):64647.

5. Zhang $P$, Wang L, Bao H, Gao Z, Chen X, Zhang D et al. Incidence of needlestick and other sharp object injuries in a Chinese hospital. Am J Infect Control. 2014; 42(2):213-4.

6. Yoshikawa T, Wada K, Lee J], Mitsuda T, Kidouchi K, Kurosu H et al. Incidence rate of needlestick and sharps injuries in 67 Japanese hospitals: a national surveillance study. PLoS One. 2013;30;8(10):e77524.

7. São Paulo. Secretaria de Saúde. Centro de Vigilância Epidemiológica. AIDS. Biológico. Boletim Epidemiológico - CRTDST/AIDS. CVE. 2012;29(1)1-116.

8. Rohde KA, Dupler AE, Postma J, Sanders A. Minimizing nurses' risks for needlestick injuries in the hospital setting. Workplace Health Saf. 2013;61(5):197-202.

9. Serdar T, Derek L, Unić A, Marijancević D, Marković D, Primorac A et al. Occupational exposures in healthcare workers in University Hospital Dubrava--10 year follow-up study. Cent Eur J Public Health. 2013 Sep;21(3):150-4.

10. Valim MD, Marziale MHP, Hayashida M, Richart-Martínez M. Ocorrência de acidentes de trabalho com material biológico potencialmente contaminado em enfermeiros. Acta Paul Enferm. 2014; 27(3):280-6.

11.Siegel JD, Rhinehart E, Jackson M, Chiarello L. Guidelines for Isolation Precautions: preventing transmission of infectious agents in healthcare settings. Am J Infect Control.

2007;35(Suppl):10:65-164).

12. Centers for Disease Control and Prevention (CDC). CDC grand rounds: preventing unsafe injection practices in the U.S. health-care system. MMWR Morb Mortal Wkly Rep. 2013 May $31 ; 62(21): 423-5$.

13. Atif ML, Brenet A, Hageaux S, Fave MH, Cochet C, Baticle E et al. Awareness of standard precautions for 4439 healthcare professionals in 34 institutions in France. Med Mal Infect. 2013; 43(1):10-6.

14. Pereira FMV, Malaguti-Toffano SE, Silva AM, Canini SRMS, Gir E. Adesão às precauções-padrão por profissionais de enfermagem que atuam em terapia intensiva em um hospital universitário. Rev Esc Enferm USP. 2013; 47(3):686-93 15. Brevidelli MM, Cianciarullo TI. Fatores psicossociais e organizacionais na adesão às Precauções-Padrão. Rev Saúde Publ. 2009; 43(6): 1-10.

16. Menezes JA, Bandeira CS, Quintana M, Silva JCADLE, Calvet GA, Brasil P. Impact of a single safety-engineered device on the occurrence of percutaneous injuries in a general hospital in Brazil. Am J Infect Control. 2014; 42(1): 174-7.

17. Machado MRM, Machado FA. Acidentes com material biológico em trabalhadores de enfermagem do Hospital Geral de Palmas (TO). Rev. Bras. Saúde Ocup. 2011;36: 124, 274-281. 18. Pimenta FR, Ferreira MD, Gir E, Hayashida M, Canini SRMS. Atendimento e seguimento clínico especializado de profissionais adesão às PP com a adesão dos profissionais na prática assistencial.

de enfermagem acidentados com material biológico. Rev Esc Enferm USP. 2013; 47(1):198-204.

19. Marziale MHP, Santos HEC, Cenzi CM, Rocha FLR, Trovó MEM. Consequências da exposição ocupacional a material biológico entre trabalhadores de um hospital universitário. Esc. Anna Nery. 2014; 18(1): 11-6.

20. Chiodi MB, Marziale MHP, Mondadori RM, Robazzi MLCC. Acidentes registrados no Centro de Referência em Saúde do Trabalhador de Ribeirão Preto. Rev. Gaúcha Enferm. 2010; 31(2): 211-217.

21. Valim MD, Marziale MHP, Hayashida M, Richart-Martínez M. Ocorrência de acidentes de trabalho com material biológico potencialmente contaminado em enfermeiros. Acta Paul. Enferm. 2014; 27(3): 280-286.

22. Canini SRMS, Moraes AS, Gir E, Freitas ICM. Fatores associados a acidentes percutâneos na equipe de enfermagem de um hospital universitário de nível terciário. Rev Lat Am Enfermagem. 2008; 16(5):818-23.

Artigo recebido em 28/06/2013.

Aprovado para publicação em 10/09/2014.

Artigo publicado em 31/03/2015. 